Bulletin of the Section of Logic

Volume 49/3 (2020), pp. 213-230

http://dx.doi.org/10.18778/0138-0680.2020.16

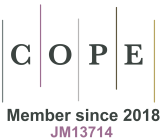

Lew Gordeev*

Edward Hermann Haeusler

\title{
PROOF COMPRESSION AND NP VERSUS PSPACE II ${ }^{1}$
}

\begin{abstract}
We upgrade [3] to a complete proof of the conjecture NP $=$ PSPACE that is known as one of the fundamental open problems in the mathematical theory of computational complexity; this proof is based on [2]. Since minimal propositional logic is known to be PSPACE complete, while PSPACE to include NP, it suffices to show that every valid purely implicational formula $\rho$ has a proof whose weight (= total number of symbols) and time complexity of the provability involved are both polynomial in the weight of $\rho$. As is [3], we use proof theoretic approach. Recall that in [3] we considered any valid $\rho$ in question that had (by the definition of validity) a "short" tree-like proof $\pi$ in the Hudelmaier-style cutfree sequent calculus for minimal logic. The "shortness" means that the height of $\pi$ and the total weight of different formulas occurring in it are both polynomial in the weight of $\rho$. However, the size (= total number of nodes), and hence also the weight, of $\pi$ could be exponential in that of $\rho$. To overcome this trouble we embedded $\pi$ into Prawitz's proof system of natural deductions containing single formulas, instead of sequents. As in $\pi$, the height and the total weight of different formulas

\footnotetext{
*Corresponding author.

${ }^{1}$ Editorial remark. The subeditor dealing with this paper (Peter SchroederHeister) and the two reviewers were not able to check proofs in all detail and therefore cannot fully confirm their correctness. However, in view of the importance of the results claimed and the originality of the logical proof methods employed, and in accordance with the aim of the journal as a forum for the wide dissemination of original results by rapid publication, they agree that the paper should be available to the scientific community in published form to enable further discussion.
}

Presented by: Peter Schroeder-Heister

Received: November 13, 2019

Published online: August 15, 2020

(C) Copyright for this edition by Uniwersytet Łódzki, Łódź 2020 
of the resulting tree-like natural deduction $\partial_{1}$ were polynomial, although the size of $\partial_{1}$ still could be exponential, in the weight of $\rho$. In our next, crucial move, $\partial_{1}$ was deterministically compressed into a "small", although multipremise, dag-like deduction $\partial$ whose horizontal levels contained only mutually different formulas, which made the whole weight polynomial in that of $\rho$. However, $\partial$ required a more complicated verification of the underlying provability of $\rho$. In this paper we present a nondeterministic compression of $\partial$ into a desired standard dag-like deduction $\partial_{0}$ that deterministically proves $\rho$ in time and space polynomial in the weight of $\rho^{2}$ Together with [3] this completes the proof of NP $=$ PSPACE.

Natural deductions are essential for our proof. Tree-to-dag horizontal compression of $\pi$ merging equal sequents, instead of formulas, is (possible but) not sufficient, since the total number of different sequents in $\pi$ might be exponential in the weight of $\rho$ - even assuming that all formulas occurring in sequents are subformulas of $\rho$. On the other hand, we need Hudelmaier's cutfree sequent calculus in order to control both the height and total weight of different formulas of the initial tree-like proof $\pi$, since standard Prawitz's normalization although providing natural deductions with the subformula property does not preserve polynomial heights. It is not clear yet if we can omit references to $\pi$ even in the proof of the weaker result NP $=$ coNP.

Keywords: Natural deduction, sequent calculus, minimal logic, computational complexity.

\section{Introduction}

In [3] we presented a dag-like version of Prawitz's [9] tree-like natural deduction calculus for minimal logic, $\mathrm{NM}_{\rightarrow}$, and left open a problem of computational complexity of the dag-like provability involved ([3, Problem 22]). In this paper we show a solution that proves the conjecture NP $=$ PSPACE. To explain it briefly first consider standard notion of provability. Recall that our basic deduction calculus $\mathrm{NM}_{\rightarrow}$ includes two basic inferences

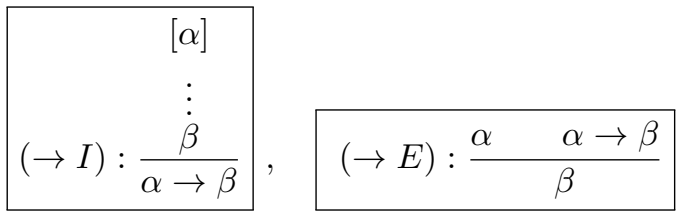

\footnotetext{
${ }^{2}$ It is doubtful that $\partial$ is convertible into $\partial_{0}$ by a polynomial-time deterministic TM.
} 
and one auxiliary repetition rule $(R): \frac{\alpha}{\alpha}$, where $[\alpha]$ in $(\rightarrow I)$ indicates that all $\alpha$-leaves occurring above $\beta$-node exposed are discharged assumptions.

Definition 1.1. A given (whether tree- or dag-like) $\mathrm{NM}_{\rightarrow}$-deduction $\partial$ proves its root-formula $\rho$ (abbr.: $\partial \vdash \rho$ ) iff every maximal thread connecting the root with a leaf labeled $\alpha$ is closed (= discharged), i.e. it contains a $(\rightarrow I)$ with conclusion $\alpha \rightarrow \beta$, for some $\beta$. A purely implicational formula $\rho$ is valid in minimal logic iff there exists a tree-like $\mathrm{NM}_{\rightarrow}$-deduction $\partial$ that proves $\rho ;^{3}$ such $\partial$ is called a proof of $\rho$.

Remark 1.2. Tree-like constraint in the definition of validity is inessential.

That is, for any dag-like $\partial \in \mathrm{NM}_{\rightarrow}$ with root-formula $\rho$, if $\partial \vdash \rho$ then $\rho$ is valid in minimal logic. Because any given dag-like $\partial$ can be unfolded into a tree-like deduction $\partial^{\prime}$ by straightforward thread-preserving bottomup recursion. To this end every node $x \in \partial$ with $n>1$ distinct conclusions has to be replaced by $n$ distinct nodes $x_{1}, \cdots, x_{n} \in \partial^{\prime}$ with corresponding single-node conclusions and identical premises of $x$. This operation obviously preserves the closure of threads, i.e. $\partial \vdash \rho$ infers $\partial^{\prime} \vdash \rho$.

Formal verification of the assertion $\partial \vdash \rho$ is simple, as follows - whether for tree-like or generally dag-like $\partial$. Every node $x \in \partial$ is assigned, by descending recursion, a set of assumptions $A(x)$ such that:

1. $A(x):=\{\alpha\}$ if $x$ is a leaf labeled $\alpha$,

2. $A(x):=A(y)$ if $x$ is the conclusion of $(R)$ with premise $y$,

3. $A(x):=A(y) \backslash\{\alpha\}$ if $x$ is the conclusion of $(\rightarrow I)$ with label $\alpha \rightarrow \beta$ and premise $y$,

4. $A(x):=A(y) \cup A(z)$ if $x$ is the conclusion of $(\rightarrow E)$ with premises $y, z$.

This easily yields

Lemma 1.3. Let $\partial \in \mathrm{NM}_{\rightarrow}$ (whether tree- or dag-like). Then $\partial \vdash \rho \Leftrightarrow$ $A(r)=\emptyset$ holds with respect to standard set-theoretic interpretations of $\cup$

${ }^{3}$ Equivalently: $\rho$ is valid in minimal logic iff it is deducible in Hilbert-style calculus with axioms $\alpha \rightarrow(\beta \rightarrow \alpha), \quad(\alpha \rightarrow(\beta \rightarrow \gamma)) \rightarrow((\alpha \rightarrow \beta) \rightarrow(\alpha \rightarrow \gamma))$ and inference $(\rightarrow E)$, also known as modus (ponendo) ponens; the equivalence follows from corresponding deduction theorem. 
and $\backslash$ in $A(r)$, where $r$ and $\rho$ are the root and root-formula of $\partial$, respectively. Moreover, $A(r) \stackrel{?}{=} \emptyset$ is verifiable by a deterministic $T M$ in $|\partial|-$ polynomial time, where by $|\partial|$ we denote the weight of (i.e. total number of symbols occurring in) $\partial .^{4}$

Now let us upgrade $\mathrm{NM}_{\rightarrow}$ to $\mathrm{NM}_{\rightarrow}^{b}$ by adding a new separation rule $(S)$

$$
(\rightarrow S): \overbrace{\frac{\overbrace{\alpha \quad \cdots}^{n \text { times }} \alpha}{\alpha}}^{(n \text { arbitrary })}
$$

whose identical premises are understood disjunctively: "if at least one premise is proved then so is the conclusion" (in contrast to ordinary conjunctive inference: "if all premises are proved then so is the conclusion"). Note that in dag-like deductions the nodes might have several conclusions (unlike in tree-like ones). The modified assignment $A$ in $\mathrm{NM}_{\rightarrow}^{b}$ (that works in both tree-like and dag-like cases) is defined by adding to old recursive clauses 1-4 (see above) a new clause 5 with new separation symbol (S):

5. $A(x)=$ S $\left(A\left(y_{1}\right), \cdots, A\left(y_{n}\right)\right)$ if $x$ is the conclusion of $(S)$ with premises $y_{1}, \cdots, y_{n}$.

Claim 1.4. For any dag-like deduction $\partial \in \mathrm{NM}_{\rightarrow}^{b}$ whose root $r$ is labeled $\rho$, $\rho$ is valid in minimal logic, provided that $A(r)$ reduces to $\emptyset$ (abbr.: $A(r) \triangleright$ $\emptyset$ ) by standard set-theoretic interpretations of $\cup, \backslash$ and nondeterministic disjunctive valuation $(S)\left(t_{1}, \cdots, t_{n}\right):=t_{i}$, for any chosen $i \in\{1, \cdots, n\}$. Moreover, the assertion $A(r) \triangleright \emptyset$ (that is also referred to as ' $\partial$ proves $\rho$ ') can be confirmed by a nondeterministic TM in $|\partial|$-polynomial time.

This claim reduces to its trivial $\mathrm{NM}_{\rightarrow}$ case (see above). For suppose that $A(r) \triangleright \emptyset$ holds with respect to a successive nondeterministic valuation of the occurrences (S). This reduction determines a successive ascending (i.e. bottom-up) thinning of $\partial$ that results in a "cleansed" $(S)$-free daglike deduction $\partial_{0} \in \mathrm{NM}_{\rightarrow}^{\mathrm{b}}$, while $A(r) \triangleright \emptyset$ in $\partial$ implies $A(r)=\emptyset$ in $\partial_{0}$. Since $(S)$ does not occur in $\partial_{0}$ anymore, we have $\partial_{0} \in \mathrm{NM}_{\rightarrow}$. By previous considerations with regard to $\mathrm{NM}_{\rightarrow}$ we conclude that $\rho$ is valid in minimal logic, which can be confirmed in $|\partial|$-polynomial time, as required.

\footnotetext{
${ }^{4}$ The latter is completely analogous to the well-known polynomial-time decidability of the circuit value problem (see also Appendix).
} 
Since minimal logic is PSPACE complete ([11, 12]), in order to arrive at the desired conclusion NP $=$ PSPACE it will suffice to show that for any valid $\rho$ there is a modified dag-like deduction $\partial \in \mathrm{NM}_{\rightarrow}^{\mathrm{b}}$ of $\rho$ satisfying $A(r) \triangleright \emptyset$, and hence a dag-like deduction $\partial_{0} \in \mathrm{NM}_{\rightarrow}$ satisfying $A(r)=\emptyset$, whose size and maximal formula weight are polynomial in $|\rho|$. But this is a consequence of [3] that formalized basic theory of dag-like deducibility in question (elaborated by the first author). For in [3] we presented a deterministic tree-to-dag horizontal compression of a given "short" tree-like deduction of $\rho$ in $\mathrm{NM}_{\rightarrow}$ that is obtained by embedding a derivation of $\rho$ in a Hudelmaier-style [5] cutfree sequent calculus. It resulted in a suitable $|\rho|$-polynomial dag-like deduction frame together with a $|\rho|$-exponential locally coherent set of maximal threads, in the multipremise expansion of $\mathrm{NM}_{\rightarrow}$ (called $\mathrm{NM}_{\rightarrow}^{*}$; multiple premises involved arise by merging equal conclusions of different rules). In this paper we observe that such a pair determines a deduction in $\mathrm{NM}_{\rightarrow}^{\mathrm{b}}$ that admits a fundamental set of chains (see below). Moreover, we show that such $\mathrm{NM}_{\rightarrow}^{b}$-deduction is convertible by the appropriate nondeterministic dag-to-dag horizontal cleansing into the required $\mathrm{NM}_{\rightarrow}$ deduction satisfying $A(r)=\emptyset .^{5}$

\subsection{Recollection of $[3]$}

Recall that $\rho$ is called dag-like provable in $\mathrm{NM}_{\rightarrow}^{*}$ iff there is a locally correct (with respect to inferences of $\mathrm{NM}_{\rightarrow}^{*}$ ) labeled regular $\operatorname{dag} \widetilde{D}=\left\langle D, \mathrm{~s}, \ell^{\mathrm{F}}\right\rangle$ (that may have arbitrary many premises and/or conclusions) with rootformula $\rho$, together with a locally coherent mapping $G: \overrightarrow{\mathrm{E}}(D) \rightarrow\{0,1\}$ that determines a set of threads that confirms alleged validity of $\rho$, where $\overrightarrow{\mathrm{E}}(D)$ denotes the set of edge-chains in $D$ (see reference in Lemma 5 below). Such $\widetilde{D}$ and a pair $\partial=\langle\widetilde{D}, G\rangle$ are called respectively a deduction frame (or just $\mathrm{NM}_{\rightarrow}^{*}$-deduction) and a dag-like proof of $\rho$ in $\mathrm{NM}_{\rightarrow \rightarrow}^{*}{ }^{6}$ In [3] we proved that the latter notion of dag-like provability of $\rho$ is equivalent to the validity of $\rho$ in minimal logic (cf. Definition 1). Without loss of generality we assume that $\widetilde{D}$ is horizontally compressed, i.e. $\ell^{\mathrm{F}}(x) \neq \ell^{\mathrm{F}}(y)$ for all $x \neq y$ on the same level in $D$, and the weight of $\widetilde{D}$ is polynomial in $|\rho|$ (see [3] and below). Such compression runs by bottom-up recursion

\footnotetext{
${ }^{5}$ This yields a "short" certificate for the local coherence statement that itself requires exponentially many bits to even describe (cf. [1, 4.3.2]).

${ }^{6}$ Here and below basic notions and notations are imported from [3].
} 
on the height of a given "short" tree-like deduction with root-formula $\rho$ by successively merging all nodes with identical formulas occurring in the corresponding horizontal sections; thus the weight of resulting dag-like deduction is polynomial in $|\rho|$, since so are the height of, and total weight of different formulas occurring in, the "short" tree-like $\mathrm{NM}_{\rightarrow}$-input in question ( [3]: Ch. 3). We noticed that the local correctness of $\widetilde{D}$ is verifiable in $|\rho|$-polynomial time, whereas the local coherence of $G$ has no obvious low-complexity upper bound, as $\overrightarrow{\mathrm{E}}(D)$ is generally exponential (cf. footnote 5$)$. The currently proposed upgrade is based on the fundamental sets of threads, instead of $G$ and $\overrightarrow{\mathrm{E}}(D)$, as follows.

\subsection{Upgrade in $\mathrm{NM}_{\rightarrow}^{*}$}

Let $\widetilde{D}=\left\langle D, \mathrm{~S}, \ell^{\mathrm{F}}\right\rangle$ be a given locally correct deduction frame with rootformula $\rho=\ell^{\mathrm{F}}(r), \mathrm{K}(D)$ be the set of maximal ascending chains (also called threads) consisting of nodes (vertices) $u \in \mathrm{V}(D)$ connecting root $r$ with leaves. A given set $\mathcal{F} \subset \mathrm{K}(D)$ is a fundamental set of threads (abbr.: $f s t)$ in $\widetilde{D}$ if the following three conditions are satisfied, where for any $\Theta=$ $\left[r=x_{0}, \cdots, x_{h(D)}\right] \in \mathrm{K}(D)$ and $i \leq h(D)$ we let $\Theta \uparrow_{x_{i}}:=\left[x_{0}, \cdots, x_{i}\right]$.

1. $\mathcal{F}$ is dense in $D$, i.e. $(\forall u \in \mathrm{V}(D))(\exists \Theta \in \mathcal{F})(u \in \Theta)$.

2. Every $\Theta \in \mathcal{F}$ is closed, i.e. its leaf-formula $\ell^{\mathrm{F}}\left(x_{h(D)}\right)$ is discharged in $\Theta$.

3. $\mathcal{F}$ preserves $(\rightarrow E)$, i.e.

$$
\begin{aligned}
& (\forall \Theta \in \mathcal{F})(\forall u \in \Theta)(\forall v \neq w \in \mathrm{V}(D):\langle u, v\rangle,\langle u, w\rangle \in \mathrm{E}(D) \wedge v \in \Theta) \\
& \left(\exists \Theta^{\prime} \in \mathcal{F}\right)\left(w \in \Theta^{\prime} \wedge \Theta \uparrow_{u}=\Theta^{\prime} \uparrow_{u}\right)
\end{aligned}
$$

LEMmA 1.5. Let $\widetilde{D}$ be as above and suppose that there exists a fst $\mathcal{F}$ in $\widetilde{D}$. Then $\rho$ is dag-like provable in $\mathrm{NM}_{\rightarrow}^{*}$.

Proof: Define $G: \overrightarrow{\mathrm{E}}(D) \rightarrow\{0,1\}$ by $G(\vec{e}):=1$ iff $(\exists \vec{f} \supseteq \vec{e}) \Theta[\vec{f}] \in$ $\mathrm{K}(D) \cap \mathcal{F}$, where $\Theta[\vec{f}]$ contains all nodes occurring in the canonical thread-expansion of $\vec{f}$. Then $\partial=\langle\widetilde{D}, G\rangle$ is a dag-like proof of $\rho$. The 
local coherence conditions 1, 2, 4, 5 (cf. [3]: Definition 6) are easily verified. In particular, 4 follows from the third $f s t$ condition with respect to $\mathcal{F}$.

Lemma 1.6. For any dag-like proof $\langle\widetilde{D}, G\rangle$ of $\rho$ there are $D_{0} \subseteq D, G_{0}$ : $\overrightarrow{\mathrm{E}}\left(D_{0}\right) \rightarrow\{0,1\}, \mathcal{F} \subset \mathrm{K}\left(D_{0}\right)$ and a dag-like proof $\left\langle\widetilde{D_{0}}, G_{0}\right\rangle$ of $\rho$ such that $\mathcal{F}$ is a fst in $\widetilde{D_{0}}$.

Proof: Let $\mathcal{F}:=\{\Theta \in \mathrm{K}(D): G(\vec{e}[\Theta])=1\}$ for $\vec{e}[\Theta]:=\overrightarrow{e_{m}} \in \overrightarrow{\mathrm{E}}(D)$ determined by $\Theta$ as specified in [3]: Definition 8. It is readily seen that such $\mathcal{F}$ is a fst in $\widetilde{D}$. The crucial condition 3 follows directly from the corresponding local coherence condition 4 (cf. [3]: Definition 6). Let $D_{0} \subseteq$ $D$ be the minimum sub-dag containing every edge occurring in $\bigcup_{\Theta \in \mathcal{F}} \Theta$ and let $\widetilde{D_{0}}=\left\langle D_{0}, \mathrm{~s}, \ell^{\mathrm{F}}\right\rangle$ be the corresponding sub-frame of $\widetilde{D}$. Obviously $\widetilde{D_{0}}$ is locally correct. Define $G_{0}: \overrightarrow{\mathrm{E}}\left(D_{0}\right) \rightarrow\{0,1\}$ as in the previous lema with respect to $D_{0}$, instead of $D$. Then $\partial=\left\langle\widetilde{D_{0}}, G_{0}\right\rangle$ is a dag-like proof of $\rho$. The crucial density of $\mathcal{F}$ in $D_{0}$ obviously follows from definitions of $D_{0}$ and $G_{0}$, as every edge in $D_{0}$ occurs in some thread from $\mathcal{F}$, while for any $\vec{e} \in \overrightarrow{\mathrm{E}}\left(D_{0}\right)$ we have $G_{0}(\vec{e})=1$ iff $\Theta[\vec{e}] \in \mathcal{F}$.

Together with [3]: Corollaries 15, 20 these lemmata yield

Corollary 1.7. Any given $\rho$ is valid in minimal logic iff there exists a pair $\langle\widetilde{D}, \mathcal{F}\rangle$ such that $\widetilde{D}$ is a locally correct deduction frame with root-formula $\rho=\ell^{\mathrm{F}}(r)$ and $\mathcal{F}$ being a fst in $\widetilde{D}$. We can just as well assume that $\widetilde{D}$ is horizontally compressed and its weight is polynomial in that of $\rho$.

Remark 1.8. We can't afford $\mathcal{F}$ to be polynomial in $\rho$. However, the existence of $\mathcal{F}$ enables a nondeterministic polytime verification of $A(r) \triangleright \emptyset$ in the corresponding modified dag-like formalism, as follows. This collapsing makes the trick.

\section{Modified dag-like calculus $\mathbf{N M}_{\rightarrow}^{b}$}

As mentioned above, our modified dag-like deduction calculus, $\mathrm{NM}_{\rightarrow}^{\mathrm{b}}$, includes inference rules $(\rightarrow I),(\rightarrow E),(R),(S)$ (see Introduction). $(\rightarrow I)$, $(R)$ and $(\rightarrow E)$ have one and two premises, respectively, whereas $(S)$ has 
two or more ones. $\mathrm{NM}_{\rightarrow}^{\mathrm{b}}$-deductions are graphically interpreted as labeled rooted regular dags (abbr.: redags, cf. [3]) $\partial=\langle\mathrm{V}(\partial), \mathrm{E}(\partial)\rangle$, whose nodes may have arbitrary many parents (conclusions) - and children (premises), just in the case $(S)$, - if any at all. The nodes $(x, y, z, \ldots)$ are labeled by $\ell^{\mathrm{F}}$ with purely implicational formulas $(\alpha, \beta, \gamma, \rho, \ldots)$. For the sake of brevity we'll assume that nodes $x$ are supplied with auxiliary height numbers $h(x) \in \mathbb{N}$, while all inner nodes also have special labels $\ell^{\mathrm{N}}(x) \in\{\mathrm{I}, \mathrm{E}, \mathrm{R}, \mathrm{S}\}$ showing the names of the inference rules $(\rightarrow I),(\rightarrow E)$, $(R),(S)$ with conclusion $x$. The roots and root-formulas are always designated $r$ and $\rho:=\ell^{\mathrm{F}}(r)$, respectively. The edges $\langle x, y\rangle \in \mathrm{E}(\partial) \subset \mathrm{V}(\partial)^{2}$ are directed upwards (thus $r$ is the lowest node in $\partial$ ) in which $x$ and $y$ are called parents and children of each other, respectively. The leaves $\mathrm{L}(\partial) \subseteq \mathrm{v}(\partial)$ are the nodes without children. Tree-like $\mathrm{NM}_{\rightarrow}^{b}$-deductions are those ones whose redags are trees (whose nodes have at most one parent).

Definition 2.1. A given $\mathrm{NM}_{\rightarrow}^{b}$-deduction $\partial$ is locally correct if conditions 1-2 are satisfied, for arbitrary nodes $x, y, z, u$.

1. $\partial$ is regular (cf. [3]), i.e.

(a) if $\langle x, y\rangle \in \mathrm{E}(\partial)$ then $x \notin \mathrm{L}(\partial)$ and $y \neq r$,

(b) $h(r)=0$,

(c) if $\langle x, y\rangle,\langle x, z\rangle \in \mathrm{E}(\partial)$ then $h(y)=h(z)=h(x)+1$.

2. $\partial$ formalizes the inference rules, i.e.

(a) if $\ell^{\mathrm{N}}(x)=\mathrm{R}$ and $\langle x, y\rangle,\langle x, z\rangle \in \mathrm{E}(\partial)$ then $y=z$ and $\ell^{\mathrm{F}}(y)=$ $\ell^{\mathrm{F}}(x)[:$ rule $(R)]$,

(b) if $\ell^{\mathrm{N}}(x)=\mathrm{I}$ and $\langle x, y\rangle,\langle x, z\rangle \in \mathrm{E}(\partial)$ then $y=z$ and $\ell^{\mathrm{F}}(x)=$ $\alpha \rightarrow \ell^{\mathrm{F}}(y)$ for some (uniquely determined) $\alpha$ [: rule $\left.(\rightarrow I)\right]$,

(c) if $\ell^{\mathrm{N}}(x)=\mathrm{E}$ and $\langle x, y\rangle,\langle x, z\rangle,\langle x, u\rangle \in \mathrm{E}(\partial)$ then $|\{y, z, u\}|=2$ and if $y \neq z$ then either $\ell^{\mathrm{F}}(z)=\ell^{\mathrm{F}}(y) \rightarrow \ell^{\mathrm{F}}(x)$ or else $\ell^{\mathrm{F}}(y)=$ $\ell^{\mathrm{F}}(z) \rightarrow \ell^{\mathrm{F}}(x)$ [: rule $\left.(\rightarrow E)\right]$,

(d) if $\ell^{\mathrm{N}}(x)=\mathrm{S}$ and $\langle x, y\rangle \in \mathrm{E}(\partial)$ then $\ell^{\mathrm{F}}(y)=\ell^{\mathrm{F}}(x)$ and $\ell^{\mathrm{N}}(y) \neq \mathrm{S}$ [: rule $(S)]$.

$\mathrm{NM}_{\rightarrow}^{*}$ is easily embeddable into $\mathrm{NM}_{\rightarrow}^{b}$. Namely, consider a locally correct $\mathrm{NM}_{\rightarrow}^{*}$-deduction frame $\widetilde{D}=\left\langle D, \mathrm{~s}, \ell^{\mathrm{F}}\right\rangle \cdot{ }^{7}$ The corresponding locally

\footnotetext{
${ }^{7}$ For brevity we omit $h$, as every $h(x)$ is uniquely determined by $x$.
} 
correct dag-like $\mathrm{NM}_{\rightarrow}^{b}$-deduction $\partial$ arises from $D$ by ascending recursion on the height. The root and basic configurations of types $(\rightarrow I),(\rightarrow E)$, $(R)$ in $\widetilde{D}$ should remain unchanged. Furthermore, if $x$ has several groups of premises in $D$, i.e. $|\mathrm{s}(x, D)|>1$ (cf. [3]) then in $\partial$ we separate these groups via $(S)$ with $|\mathrm{s}(x, D)|$ identical premises; for example this multipremise $\mathrm{NM}_{\rightarrow}^{*}$-configuration in $\widetilde{D}$

$$
\frac{\frac{\beta \quad \gamma \quad \gamma \rightarrow(\alpha \rightarrow \beta)}{\alpha \rightarrow \beta}}{\gamma \rightarrow(\alpha \rightarrow \beta)}
$$

goes to this $\mathrm{NM}_{\rightarrow}^{b}$-configuration in $\partial$

$$
(\rightarrow I) \frac{(S) \frac{(\rightarrow I) \frac{\beta}{\alpha \rightarrow \beta} \quad(\rightarrow E) \frac{\gamma}{\alpha \rightarrow(\alpha \rightarrow \beta)}}{\alpha \rightarrow \beta}}{\gamma \rightarrow(\alpha \rightarrow \beta)} \text {. }
$$

Corresponding $\ell^{\mathrm{F}}$ - and $\ell^{\mathrm{N}}$-labels are induced in an obvious way. Note that the weight of $\partial$ is linear in that of $\widetilde{D}^{8}$

Now suppose that there is a $f s t \mathcal{F}$ in a chosen $\mathrm{NM}_{\rightarrow}^{*}$-deduction frame $\widetilde{D}$, and let $\mathcal{F}^{b}$ be the image of $\mathcal{F}$ in $\partial$. It is readily seen that $\mathcal{F}^{b}$ is also a dense and $(\rightarrow E)$ preserving set of closed threads in $\partial$ (see $\mathrm{NM}_{\rightarrow}^{*}$-clauses $1-3$ in Ch. 1.2). That is, $\mathcal{F}^{b}$ is a dense set of closed threads in $\partial$ such that for every $\Theta \in \mathcal{F}^{b}$ and $(\rightarrow E)$-conclusion $x \in \Theta, \ell^{\mathrm{N}}(x)=\mathrm{E}_{\rightarrow}$, with premises $y$ and $z$, if $y \in \Theta$ then there is a $\Theta^{\prime} \in \mathcal{F}^{b}$ such that $z \in \Theta^{\prime}$ and $\Theta$ coincides with $\Theta^{\prime}$ below $x$.

\subsection{Modified dag-like provability}

We formalize in $\mathrm{NM}_{\rightarrow}^{b}$ the modified assignment $\mathcal{A}: \partial \ni x \hookrightarrow A(x) \subseteq$ $\operatorname{FOR}(\partial)$.

Definition 2.2 (Assignment $\mathcal{A}$ ). Let $\partial$ be any locally correct dag-like $\mathrm{NM}_{\rightarrow}^{b}$-deduction. We assign nodes $x \in \partial$ with terms $A(x)$ by descending recursion $1-5$.

\footnotetext{
${ }^{8}$ Recall that according to [3] we can just as well assume that $\widetilde{D}$ is horizontally compressed and its weight is polynomial in that of $\rho$.
} 
1. $A(x):=\{\alpha\}$ if $x$ is a leaf and $\ell^{\mathrm{F}}(x)=\alpha$.

2. $A(x):=A(y)$ if $\ell^{\mathrm{N}}(x)=\mathrm{R}$ and $\langle x, y\rangle \in \mathrm{E}(\partial)$.

3. $A(x):=A(y) \backslash\{\alpha\}$ if $\ell^{\mathrm{N}}(x)=\mathrm{I},\langle x, y\rangle \in \mathrm{E}(\partial)$ and $\ell^{\mathrm{F}}(x)=\alpha \rightarrow \ell^{\mathrm{F}}(y)$.

4. $A(x):=A(y) \cup A(z)$ if $\ell^{\mathrm{N}}(x)=\mathrm{E}$ and $\langle x, y\rangle,\langle x, z\rangle \in \mathrm{E}(\partial)$.

5. $A(x):=\mathrm{S}\left(A\left(y_{1}\right), \cdots, A\left(y_{n}\right)\right)$ if $\ell^{\mathrm{N}}(x)=\mathrm{S}$ and $(\forall i \in[1, n])\left\langle x, y_{i}\right\rangle \in$ $\mathrm{E}(\partial)$.

Definition 2.3 (Nondeterministic reduction). Let $\partial$ and $\mathcal{A}$ be as above, $r$ the root of $\partial, S$ a set of formulas occurring in $\partial$. We say that $A(r)$ reduces to $S$ (abbr.: $A(r) \triangleright S$ ) if $S$ arises from $A(r)$ by successive (in a left-toright direction) substitutions $A(u)=\$\left(A\left(v_{1}\right), \cdots, A\left(v_{n}\right)\right):=A\left(v_{i}\right)$, for a fixed chosen $i \in\{1, \cdots, n\}$ and for any occurrence $A(u)$ in $A(w)$ and in $A\left(w^{\prime}\right)$, for every $w^{\prime}$ below $w$, provided that $u$ is a premise of $w$ such that $\ell^{\mathrm{N}}(u)=\mathrm{s},{ }^{9}$ while using ordinary set-theoretic interpretations of $\cup$ and $\backslash$. We call $\partial$ a modified dag-like proof of $\rho=\ell^{\mathrm{F}}(r)($ abbr.: $\partial \vdash \rho)$ if $A(r) \triangleright \emptyset$ holds. ${ }^{10}$

Example 2.4. Previously shown configuration yields a $\partial$ such that $\partial \nvdash \rho$ :

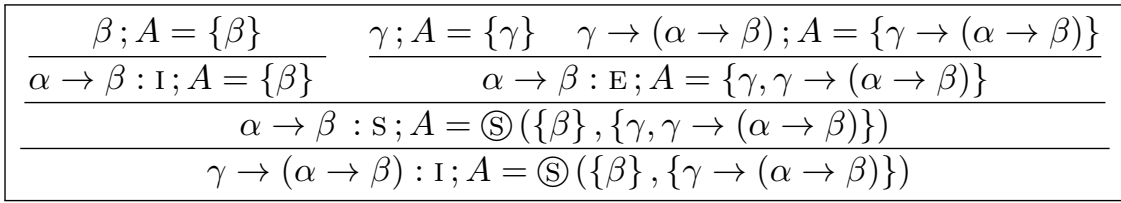

where $\ell^{\mathrm{N}}(r)=\mathrm{I}, \quad \ell^{\mathrm{F}}(r)=\rho=\gamma \rightarrow(\alpha \rightarrow \beta)$ and $A(r)=\mathrm{S}(\{\beta\}$, $\{\gamma \rightarrow(\alpha \rightarrow \beta)\})$. Note that $A(r) \triangleright\{\beta\}$ and $A(r) \triangleright\{\gamma \rightarrow(\alpha \rightarrow \beta)\}$, although $A(r) \not \emptyset$.

To obtain an analogous dag-like proof of (say) $\rho^{\prime}:=\beta \rightarrow(\gamma \rightarrow(\alpha \rightarrow \beta))$ we'll upgrade $\partial$ to such $\partial^{\prime}$ :

${ }^{9}$ This operation is graphically interpreted by deleting $u$ along with $v_{j}$ for all $j \neq i$.

${ }^{10}$ The nondeterminism in question is encoded in (S) of Clause 5. 


\begin{tabular}{|cc|}
$\frac{\frac{\beta ; A=\{\beta\}}{\alpha \rightarrow \beta: \mathrm{I} ; A=\{\beta\}}}{\frac{\gamma ; A=\{\gamma\} \quad \gamma \rightarrow(\alpha \rightarrow \beta) ; A=\{\gamma \rightarrow(\alpha \rightarrow \beta)\}}{\alpha \rightarrow \beta: \mathrm{E} ; A=\{\gamma, \gamma \rightarrow(\alpha \rightarrow \beta)\}}}$ \\
$\frac{\alpha \rightarrow \beta: \mathrm{S} ; A=\mathrm{S}(\{\beta\},\{\gamma, \gamma \rightarrow(\alpha \rightarrow \beta)\})}{\gamma \rightarrow(\alpha \rightarrow \beta): \mathrm{I} ; A=\mathrm{S}(\{\beta\},\{\gamma \rightarrow(\alpha \rightarrow \beta)\}) \backslash\{\gamma\}}$ \\
\hline$\beta \rightarrow(\gamma \rightarrow(\alpha \rightarrow \beta)): \mathrm{I} ; A=\mathrm{S}(\{\beta\},\{\gamma \rightarrow(\alpha \rightarrow \beta)\}) \backslash\{\gamma\} \backslash\{\beta\}$
\end{tabular}

and let $S(\{\beta\},\{\gamma, \gamma \rightarrow(\alpha \rightarrow \beta)\}):=\{\beta\}$. Then $A(r) \triangleright \emptyset$, i.e. $\partial^{\prime} \vdash \rho^{\prime}$ holds.

LEMma 2.5. Every modified dag-like proof of $\rho$ is convertible to a dag-like $\mathrm{NM}_{\rightarrow}$-proof of $\rho$.

Proof: Let $\partial$ be a given $\mathrm{NM}_{\rightarrow}^{b}$-proof of $\rho$. Its $\mathrm{NM}_{\rightarrow}$-conversion is defined by a simple ascending recursion, as follows. Each time we arrive at a $w$ whose premise $u$ is a conclusion of $(S)$, we replace $u$ by its premise that is "guessed" by a given nondeterministic reduction leading to $A(r) \triangleright \emptyset$ - alternatively, we can replace this $(S)$ by the corresponding repetition $(R)$. It is readily seen that the resulting dag-like deduction $\partial_{0}$ with the same root-formula $\rho$ is locally correct and $(S)$-free, and hence it belongs to $\mathrm{NM}_{\rightarrow}$. Obviously $A(r) \triangleright \emptyset$ in $\partial$ infers $A(r)=\emptyset$ in $\partial_{0}$, and hence $\partial_{0}$ proves $\rho$ in $\mathrm{NM}_{\rightarrow}$.

This lemma is generalized by

LEMMA 2.6. Let $\widetilde{D}$ be any locally correct deduction frame in $\mathrm{NM}_{\rightarrow}^{*}$ with root-formula $\rho$ that admits some fst. There exists a dag-like $\mathrm{NM}_{\rightarrow}-$-proof of $\rho$ whose weight does not exceed that of $\widetilde{D}$.

Proof: Let $\partial$ be the $\mathrm{NM}_{\rightarrow}^{b}$-deduction of $\rho$ induced by $\widetilde{D}$ and $\mathcal{F}$ any $f s t$ in $\widetilde{D}$. Furthermore, let $\mathcal{F}^{b}$ be the image of $\mathcal{F}$ in $\partial$ (see above). We will show that $\mathcal{F}^{b}$ determines successive left-to-right (S-eliminations (S) $\left(A\left(y_{1}\right), \cdots, A\left(y_{n}\right)\right) \hookrightarrow A\left(y_{i}\right)$ inside $A(r)$ leading to a desired reduction $A(r) \triangleright \emptyset$. These eliminations together with a suitable sub-fst $\mathcal{F}_{0}^{b} \subseteq \mathcal{F}^{b}$ arise as follows by ascending recursion along $\mathcal{F}^{b}$. Let $x$ with $\ell^{\mathrm{N}}(x)=\mathrm{E}$ be a chosen lowest conclusion of $(\rightarrow E)$ in $\partial$, if any exists. By the density of $\mathcal{F}^{b}$, there exists $\Theta \in \mathcal{F}^{b}$ with $x \in \Theta$; we let $\Theta \in \mathcal{F}_{0}^{b}$. Let $y$ and $z$ be the two premises of $x$ and suppose that $y \in \Theta$. By the third fst condition there exists a $\Theta^{\prime} \in \mathcal{F}^{b}$ with $z \in \Theta^{\prime}$ and $\Theta \uparrow_{x}=\Theta^{\prime} \uparrow_{x}$; so let $\Theta^{\prime} \in \mathcal{F}_{0}^{b}$ be the corresponding "upgrade" of $\Theta$. In the case $z \in \Theta$ we let $\Theta^{\prime}:=\Theta$. Note that $\left.\Theta\right|_{x}$ 
determines substitutions $A(u)=$ (S) $\left(A\left(v_{1}\right), \cdots, A\left(v_{n}\right)\right):=A\left(v_{i}\right)$ in all parents of $(S)$-conclusions $u$ occurring in both $\Theta$ and $\Theta^{\prime}$ below $x$ (cf. Definitions 10,11$)$, if any exist, and hence also (S-eliminations $A(u) \hookrightarrow A\left(v_{i}\right)$ in the corresponding subterms of $A(r)$. The same procedure is applied to the nodes occurring in $\Theta$ and $\Theta^{\prime}$ between $x$ and the next lowest conclusions of $(\rightarrow E)$; this yields new "upgraded" threads $\Theta^{\prime \prime}, \Theta^{\prime \prime \prime}, \cdots \in \mathcal{F}_{0}^{b}$ and (S)-eliminations in the corresponding initial fragments of $A(r)$. We keep doing this recursively until the list of remaining (S-occurrences in $\Theta \in \mathcal{F}_{0}^{b}$ is empty. The final "cleansed" (S-free form of $A(r)$ is represented by a set of formulas that easily reduces to $\emptyset$ by ordinary set-theoretic interpretation of the remaining operations $\cup$ and $\backslash$, since every $\Theta \in \mathcal{F}_{0}^{b}$ involved is closed. That is, the correlated "cleansed" deduction $\partial_{0}$ is a locally correct dag-like deduction of $\rho$ in the $(S)$-free fragment of $\mathrm{NM}_{\rightarrow}^{b}$, and hence it belongs to $\mathrm{NM}_{\rightarrow}$; moreover the set of ascending threads in $\partial_{0}$ is uniquely determined by the remaining rules $(R),(\rightarrow I),(\rightarrow E)$ (cf. analogous passage in the previous proof). Now by the definition these "cleansed" ascending threads are all included in $\mathcal{F}_{0}^{b}$ and hence closed with respect to $(\rightarrow I) .{ }^{11}$ This yields a desired reduction $A(r) \triangleright \emptyset$, i.e. $A(r)=\emptyset$, in $\partial_{0}$. Hence $\partial_{0}$ proves $\rho$ in $\mathrm{NM}_{\rightarrow}$. Obviously the weight of $\partial_{0}$ does not exceed the weight of $\widetilde{D}$.

Operation $\partial \hookrightarrow \partial_{0}$ is referred to as horizontal cleansing (cf. Introduction). Together with Remark 2 and Corollary 7 this yields

Corollary 2.7. Any given $\rho$ is valid in minimal logic iff it is provable in $\mathrm{NM}_{\rightarrow}$ by a dag-like deduction $\partial_{0}$ whose weight is polynomial in $|\rho|$ and such that $\partial_{0} \vdash \rho$ can be confirmed by a deterministic TM in $|\rho|$-polynomial time. $^{12}$

\section{Theorem 2.8. PSPACE $\subseteq \mathbf{N P}$ and hence $\mathbf{N P}=$ PSPACE.}

Proof: Minimal propositional logic is PSPACE-complete (cf. e.g. [7, 11, 12]). Hence PSPACE $\subseteq \mathbf{N P}$ directly follows from Corollary 15. Note that in contrast to [3] here we use nondeterministic arguments twice. First we "guess" the existence of "short" Hudelmaier-style cutfree sequential deduction of $\rho$ that leads (by deterministic compression) to a "small" natural deduction frame $\widetilde{D}$ that is supposed to have a fst $\mathcal{F}$. Then we "guess"

\footnotetext{
${ }^{11}$ These threads may be exponential in number, but our nondeterministic algorithm runs on the polynomial set of nodes.

${ }^{12}$ See Appendix for a more exhaustive presentation.
} 
the existence of a "cleansed" modified subdeduction that confirms in $|\rho|$ polynomial time the provability of $\rho$ with regard to $\langle\widetilde{D}, \mathcal{F}\rangle$.

Corollary 2.9. $\mathbf{N P}=\mathbf{c o N P}$ and hence the polynomial hierarchy collapses to the first level.

Proof $: \mathbf{N P}=\mathbf{P S P A C E}$ implies $\operatorname{coNP}=\mathbf{c o P S P A C E}=\mathbf{P S P A C E}=\mathbf{N P}$ (see also $[8,1])$.

Corollary 2.10. PSPACE (in particular NP) problems are nondeterministically decidable in polynomial time. To put it more precisely, for any given PSPACE language $L \subseteq\{0,1\}^{*}$ there exists a polynomial $p: \mathbb{N} \rightarrow \mathbb{N}$ and a polynomial-time TM $M$ such that for every $x \in\{0,1\}^{*}$ there exists $u \in\{0,1\}^{p(|x|)}$ satisfying $x \in L \Leftrightarrow M(x, u)=1$ (i.e.: " $u$ provides $a$ polynomial test for $\left.x \in \in^{?} L^{\prime \prime}\right) .{ }^{13}$

Proof: By theorem 16, it suffices to deal with the NP-complete problem of boolean satisfiability. Let $\varphi(\vec{v})$ be a given boolean formula, where $\vec{v}$ is a list of propositional variables that is encoded by $x \in\{0,1\}^{*}$. Let $x \in L$ abbreviate $\varphi(\vec{v}) \in \mathrm{SAT}$, then $x \notin L \Leftrightarrow \neg \varphi(\vec{v}) \in$ VAL. By Corollary 17, SAT and VAL are both in NP. This yields the result by an obvious nondeterministic combination of standard NP-verifications of both conjectures $x \in L$ and $x \notin L$.

Remark 2.11 ("Hilbert-paradise" of PSPACE world). Corollary 18 yields a following broad conclusion. PSPACE problems are closed under propositional operations and provability (by Savitch's theorem) while being (nondeterministic) decidable in polynomial time (: "in PSPACE there is no polytime ignorabimus").

${ }^{13}$ That is, we rewrite NP condition

$$
\left(\forall x \in\{0,1\}^{*}\right)\left(x \in L \Leftrightarrow\left(\exists u \in\{0,1\}^{p(|x|)}\right) M(x, u)=1\right)
$$

(cf. e.g. $[1,2.1])$ to

$$
\left(\forall x \in\{0,1\}^{*}\right)\left(\exists u \in\{0,1\}^{p(|x|)}\right)(x \in L \Leftrightarrow M(x, u)=1)
$$

or, more precisely, to

$$
\left(\forall x \in\{0,1\}^{*}\right)\left(\neg \neg \exists u \in\{0,1\}^{p(|x|)}\right)(x \in L \Leftrightarrow M(x, u)=1) .
$$




\section{References}

[1] S. Arora, B. Barak, Computational Complexity: A Modern Approach, 1st ed., Cambridge University Press, USA (2009).

[2] L. Gordeev, Proof compression and NP versus PSPACE. Part 2, CoRR, vol. abs/1907.03858 (2019), URL: http://arxiv.org/abs/1907.03858.

[3] L. Gordeev, E. H. Haeusler, Proof Compression and NP Versus PSPACE, Studia Logica, vol. 107(1) (2019), pp. 53-83, DOI: http://dx.doi.org/10. 1007/s11225-017-9773-5.

[4] J. Holm, E. Rotenberg, M. Thorup, Planar Reachability in Linear Space and Constant Time, CoRR, vol. abs/1411.5867 (2014), URL: http://arxiv.org/ abs/1411.5867.

[5] J. Hudelmaier, An $O(n \log n)$-Space Decision Procedure for Intuitionistic Propositional Logic, Journal of Logic and Computation, vol. 3(1) (1993), pp. 63-75, DOI: http://dx.doi.org/10.1093/logcom/3.1.63.

[6] H. Ishihara, H. Schwichtenberg, Embedding classical in minimal implicational logic, Mathematical Logic Quarterly, vol. 62(1-2) (2016), pp. 94-101, DOI: http://dx.doi.org/10.1002/malq.201400099.

[7] I. Johansson, Der Minimalkalkül, ein reduzierter intuitionistischer Formalismus, Compositio Mathematica, vol. 4 (1937), pp. 119-136, URL: http://www.numdam.org/item/CM_1937_-4__119_0.

[8] C. H. Papadimitriou, Computational complexity, AddisonWesley (1994).

[9] D. Prawitz, Natural Deduction: A Proof-theoretical Study, Almqvist \& Wiksell (1965).

[10] D. Prawitz, P.-E. Malmnäs, A Survey of Some Connections Between Classical, Intuitionistic and Minimal Logic, [in:] H. A. Schmidt, K. Schütte, H.-J. Thiele (eds.), Contributions to Mathematical Logic, vol. 50 of Studies in Logic and the Foundations of Mathematics, Elsevier (1968), pp. 215-229, DOI: http://dx.doi.org/10.1016/S0049-237X(08)70527-5.

[11] R. Statman, Intuitionistic Propositional Logic is Polynomial-Space Complete, Theoretical Computer Science, vol. 9 (1979), pp. 67-72, DOI: http://dx.doi.org/10.1016/0304-3975(79)90006-9. 
[12] V. Svejdar, On the polynomial-space completeness of intuitionistic propositional logic, Archive for Mathematical Logic, vol. 42(7) (2003), pp. 711-716, DOI: http://dx.doi.org/10.1007/s00153-003-0179-x.

[13] M. Thorup, Compact oracles for reachability and approximate distances in planar digraphs, Journal of the ACM, vol. 51(6) (2004), pp. 993-1024, DOI: http://dx.doi.org/10.1145/1039488.1039493.

\section{Appendix: rough complexity estimate}

\section{Dag-like proof system $\mathrm{NM}_{\rightarrow}$}

We regard $\mathrm{NM}_{\rightarrow}$ as $\mathrm{NM}_{\rightarrow}^{b}$ without separation rule $(S)$. Moreover, without loss of generality we suppose that dag-like $\mathrm{NM}_{\rightarrow}$-deductions $\partial$ of rootformulas $\rho$ have polynomial total number of vertices $|\mathrm{v}(\partial)|=\mathcal{O}\left(|\rho|^{4}\right)$ while the weights of formulas and the height numbers involved are bounded by $2|\rho|$ and $|\mathrm{V}(\partial)|$, respectively (cf. [3]).

Let LC $(\partial)$ and PROV $(\partial)$ be abbreviations for ' $\partial$ is locally correct' and ' $\partial$ proves $\rho$ ', respectively, and let $\operatorname{PROOF}(\partial):=\mathrm{LC}(\partial) \& \operatorname{PROV}(\partial)$. We wish to validate the assertion $\operatorname{PROOF}(\partial)$ in polynomial time (and space) by a suitable deterministic TM $M$. For technical reasons we choose a formalization of $\partial$ in which edges are redefined as pairs $\langle$ parent, child $\rangle$. Let $\rho, \chi_{\rho} \in\{\mathrm{I}, \mathrm{E}\}, a=2|\rho|$ and $0<r<b=\mathcal{O}\left(|\rho|^{4}\right)$ be fixed.

Input of $M$ : List $\mathbf{t}$ consisting of tuples $t(x)=\left[x, y_{1}, y_{2}, h, h_{1}, h_{2}, \chi, \gamma\right.$, $\left.\beta_{1}, \beta_{2}\right]$, for all $0<x \leq b$, where $\chi \in\{\mathrm{R}, \mathrm{I}, \mathrm{E}, \mathrm{L}\}$ (L stands for 'leaf'), while $x, y_{1}, y_{2} \leq b, h, h_{1}, h_{2} \leq b$ and $\gamma, \beta_{1}, \beta_{2} \leq a$ are natural numbers (in binary) which are thought to encode nodes, nodes' heights and formulas, respectively $(0$ encodes $\emptyset$ ).

The weight of $\mathbf{t}$ is $\mathcal{O}\left(|\rho|^{4} \log |\rho|\right)<\mathcal{O}\left(|\rho|^{5}\right) . \operatorname{LC}(\partial)$ and PROV $(\partial)$ are verified by $M$ as follows while assuming that: $x$ are parents of $y_{i}>0$, $h:=h(x), h_{i}:=h\left(y_{i}\right), \gamma:=\ell^{\mathrm{F}}(x), \beta_{i}:=\ell^{\mathrm{F}}\left(y_{i}\right)(i \in\{1,2\})$ and $\chi:=\ell^{\mathrm{N}}(x)$ if $x$ is not a leaf, else $\chi:=\mathrm{L}$.

\section{Local correctness}

$\mathrm{LC}(\partial)$ is equivalent to conjunction of the following conditions $1-8$ on $\mathbf{t}$ that (according to above assumptions) uniquely determines the underlying locally correct $\mathrm{NM}_{\rightarrow}$-deduction $\partial$ by ascending induction on $h$. 
1. If $x=x^{\prime}$ then $t(x)=t\left(x^{\prime}\right)$.

2. If $t(x)=\left[x, y_{1}, y_{2}, h, h_{1}, h_{2}, \chi, \gamma, \beta_{1}, \beta_{2}\right]$ and $x^{\prime}=y_{i}>0(i \in\{1,2\})$ for $t\left(x^{\prime}\right)=\left[x^{\prime}, y_{1}^{\prime}, y_{2}^{\prime}, h^{\prime}, h_{1}^{\prime}, h_{2}^{\prime}, \chi^{\prime}, \chi_{1}^{\prime}, \chi_{2}^{\prime}, \gamma^{\prime}, \beta_{1}^{\prime}, \beta_{2}^{\prime}\right]$, then $h^{\prime}=h_{i}$ and $\gamma^{\prime}=\beta_{i}$.

3. If $x=r$ then $h=0, \gamma=\rho$ and $\chi=\chi_{\rho}$.

4. If $\chi=\mathrm{L}$ then $y_{1}=y_{2}=\beta_{1}=\beta_{2}=0$ [: case $\left.x \in \mathrm{L}(\partial)\right]$.

5. If $\chi \neq \mathrm{L}$ then $y_{1}+y_{2}>0$ and $h_{1}=h_{2}=h+1$.

6. If $\chi=\mathrm{R}$ then $y_{2}+\beta_{2}=0<y_{1}$ and $\gamma=\beta_{1}$ [: rule $\left.(R)\right]$.

7. If $\chi=\mathrm{I}$ then $\gamma=\alpha \rightarrow \beta_{1}$ (for some $\alpha$ ) [: rule $\left.(\rightarrow I)\right]$.

8. If $\chi=\mathrm{E}$ then $\beta_{2}=\beta_{1} \rightarrow \gamma[:$ rule $(\rightarrow E)]$.

The verification of conditions $1-8$ requires $\mathcal{O}\left(|\rho|^{5}\right)$ iterations of basic queries $\chi \stackrel{?}{=} \chi^{\prime}, u \stackrel{?}{=} v, \delta \stackrel{?}{=} \sigma,(\exists ? \alpha) \gamma=\alpha \rightarrow \beta$ for $\chi, \chi^{\prime} \in\{\mathrm{R}, \mathrm{I}, \mathrm{E}, \mathrm{L}\}$, $u, v \leq b$ and $\beta, \gamma, \delta, \sigma \leq a$ that are solvable in $\mathcal{O}(|\rho|)$ time (note that $\alpha \rightarrow \beta=\rightarrow \alpha \beta$ in the Eukasiewicz prefix notation). Summing up there is a deterministic TM $M$ that verifies $\operatorname{LC}(\partial)$ in $\mathcal{O}\left(|\rho|^{5} \cdot|\rho|\right)=\mathcal{O}\left(|\rho|^{6}\right)$ time and $\mathcal{O}\left(|\rho|^{5}\right)$ space.

\section{Assignment $\mathcal{A}$}

A given locally correct $\mathrm{NM}_{\rightarrow}$-deduction $\partial$ determines an assignment

$$
\mathcal{A}: 0<x \leq b \hookrightarrow A(x) \subseteq \mathrm{FOR}(\partial)
$$

that is defined by the following recursive clauses $1-4$ for input $\mathbf{t}$ satisfying above conditions $1-8$, where as above $t(x)=\left[x, y_{1}, y_{2}, h, h_{1}, h_{2}, \chi, \gamma, \beta_{1}, \beta_{2}\right]$, for all $0<x \leq b$.

1. $A(x):=\{\gamma\}$ if $\chi=\mathrm{L}$.

2. $A(x):=A\left(y_{1}\right)$ if $\chi=\mathrm{R}$.

3. $A(x):=A\left(y_{1}\right) \backslash\{\alpha\}$ if $\chi=\mathrm{I}$ and $\gamma=\alpha \rightarrow \beta_{1}$.

4. $A(x):=A\left(y_{1}\right) \cup A\left(y_{2}\right)$ if $\chi=\mathrm{E}$. 
The length of recursion $1-4$ is $b=\mathcal{O}\left(|\rho|^{4}\right)$. Recursion steps produce (say, sorted) lists of formulas $A(x),|A(x)| \leq b$ using set-theoretic unions $A \cup B$ and subtractions $A \backslash\{\alpha\}$. Each recursion step requires $\mathcal{O}(b \cdot|\rho|)=$ $\mathcal{O}\left(|\rho|^{5}\right)$ steps of computation. This yields upper bound $\mathcal{O}\left(|\rho|^{4} \cdot|\rho|^{5}\right)=$ $\mathcal{O}\left(|\rho|^{9}\right)$ for $A(r) \stackrel{?}{=} \emptyset$. Thus $\operatorname{PROV}(\partial)$ is verifiable in $\mathcal{O}\left(|\rho|^{9}\right)$ time and $\mathcal{O}(|\rho|)$ space. Hence by the above estimate of $\mathrm{LC}(\partial)$ we can safely assume that $\operatorname{PROOF}(\partial)$ is verifiable by a deterministic TM $M$ in $\mathcal{O}\left(|\rho|^{9}\right)$ time and $\mathcal{O}\left(|\rho|^{5}\right)$ space.

Conclusion 2.12. There exist polynomials $p, q, r$ of degrees $5,9,5$, respectively, and a deterministic boolean-valued TM $M$ such that for any purely implicational formula $\rho$ the following holds: $\rho$ is valid in minimal logic iff there exists a $u \in\{0,1\}^{p(|\rho|)}$ such that $M(\rho, u)$ yields 1 after $q(|\rho|+|u|)$ steps of computation in space $r(|\rho|+|u|)$. Analogous polynomial estimates of the intuitionistic and/or classical propositional and even quantified boolean validity are easily obtained by familiar syntactic interpretations within minimal logic (cf. e.g. [6, 10, 12]).

Remark 2.13. Recall that PROV $(\partial)$ is equivalent to the assertion that maximal threads in $\partial$ are closed. This in turn is equivalent to a variant of non-reachability assertion: ' $r$ is not connected to any leaf $z$ in a subgraph of $\partial$ that is obtained by deleting all edges $\langle x, y\rangle$ with $\ell^{\mathrm{N}}(x)=\mathrm{I}$ and $\ell^{\mathrm{F}}(x)=$ $\ell^{\mathrm{F}}(z) \rightarrow \ell^{\mathrm{F}}(y)^{\prime}$, which we'll abbreviate by $\mathrm{PROV}_{1}(\partial)$. Now $\operatorname{PROV}_{1}(\partial)$ is verifiable by a deterministic $\mathrm{TM}$ in $\mathcal{O}(|\mathrm{V}(\partial)| \cdot|\mathrm{E}(\partial)|)=\mathcal{O}\left(|\rho|^{12}\right)$ time and $\mathcal{O}(|\rho| \cdot|\mathrm{V}(\partial)|)=\mathcal{O}\left(|\rho|^{5}\right)$ space (cf. e.g. [8]). However this does not improve our upper bound for PROOF $(\partial)$. Actually there are known much better estimates of the reachability problem (cf. e.g. [13, 4]), but at this stage we are not interested in a more precise analysis.

\section{Lew Gordeev}

University of Tübingen

Department of Computer Science

Sand 14, 72076 Tübingen, Nedlitzer Str. 4a

14612 Falkensee, Germany

e-mail: lew.gordeew@uni-tuebingen.de 
Edward Hermann Haeusler

Pontificia Universidade Católica do Rio de Janeiro - RJ

Department of Informatics

Rua Marques de São Vicente, 224, Gávea

Rio de Janeiro, Brasil

e-mail: hermann@inf.puc-rio.br 